\title{
INFLUENCIA DE LA CONTAMINACIÓN DE LAS BALSAS Y RESTAURANTES FLOTANTES EN EL ESTANCAMIENTO DEL DESARROLLO TURÍSTICO SOSTENIBLE EN LA LAGUNA DE YARINACOCHA, REGIÓN UCAYALI- 2016.
}

\author{
INFLUENCE OF THE POLLUTION OF THE RAFTS AND FLOATING \\ RESTAURANTS IN THE STAGNATION OF THE SUSTAINABLE TOURIST \\ DEVELOPMENT IN THE YARINACOCHA LAGOON, REGION UCAYALI - 2016.
}

\author{
MG. RAFAEL A. GARAY ARGANDOÑA / PIERRE INUMA RODRIGUEZ
}

\section{RESUMEN}

Objetivo: La presente investigación busca determinar la influencia de la contaminación de las balsas y restaurantes flotantes en la laguna de Yarinacocha y el estancamiento del desarrollo turístico sostenible en la rivera de la laguna, en el Distrito de Yarinacocha, Región Ucayali- 2016. Materiales y Métodos: La investigación se llevó a cabo utilizando la metodología explicativa, se realizó en el Distrito de Yarinacocha, específicamente alrededor de la laguna de Yarinacocha, que serán desarrollados en puntos estratégicos en donde se concentra el mayor flujo de visitantes, establecimientos comerciales, y pobladores. La técnica empleada para este trabajo de investigación fue la encuesta por medio de un cuestionario. Para ello se trabajó con una muestra de 50 turistas nacionales, los cuales eran abordados al momento de llegar a la rivera de la Laguna de Yarinacocha. Resultados: Los resultados de la encuesta, muestra lo siguiente El 36 \% de los encuestados tiene una impresión regular de la laguna de Yarinacocha, de igual forma un 26\% dice que la impresión que llegan a tener es muy buena, así mismo un $24 \%$ asegura que es muy buena. Mientras de otro lado tenemos un $8 \%$ y $6 \%$ que considera que es muy mala y mala la impresión que tienen respectivamente. Conclusiones: Se concluye que el posicionamiento de las balsas y restaurantes flotantes dentro de la Laguna de Yarinacocha, afectan significativamente con el desarrollo turístico del mismo, esto imposibilita que pueda mejorar el interés en los posibles visitantes, además que es un factor clave para el desarrollo en otros aspectos que tiene una sociedad como son el económico, político, social y cultural; por la forma en como se ha desarrollado el trabajo además se puede concluir que las personas que habitan en la rivera de la laguna de Yarinacocha no son conscientes del impacto que puede generar la contaminación del agua. Recomendaciones: Se recomienda que 
las organizaciones públicas y privadas puedan implementar programas enfocados al desarrollo turístico, para ello es importante que trabajen directamente con las personas que viven en la rivera de la Laguna de Yarinacocha, dueños de establecimientos y otros; ya sea brindando charlas sobre la concientización sobre el cuidado del Agua.

Palabras Claves: Influencia, contaminación, desarrollo, turismo, estancamiento, sostenibilidad.

\begin{abstract}
Objective: The present research seeks to determine the influence of the contamination of floating rafts and restaurants in the Yarinacocha lagoon and the stagnation of sustainable tourism development on the lagoon side, in the Yarinacocha District, Ucayali- Region 2016. Materials and Methods: The research was carried out using the explanatory methodology, carried out in the Yarinacocha District, specifically around the Yarinacocha lagoon, which will be developed at strategic points where the highest flow of visitors, commercial establishments, and settlers is concentrated. The technique used for this research was the survey by means of a questionnaire. For this purpose, a sample of 50 national tourists was studied, which were approached at the time of arriving at the shore of the Laguna de Yarinacocha. Results: The results of the survey, shows that $36 \%$ of the respondents have a regular printing of Yarinacocha lagoon, in the same form $26 \%$ said that the impression that reach was very good, also $24 \%$ ensures that it is very good. While on the other hand we have a $8 \%$ and $6 \%$, which is very bad and bad print who have respectively. Conclusions: It is concluded that the positioning of floating rafts and restaurants within the Laguna de Yarinacocha, significantly affect the tourism development of the same, this makes it impossible to improve interest in potential visitors, and is a key factor for development in others Aspects that have a society as they are the economic, political, social and cultural; By the way in which the work has been developed, it can also be concluded that the people living on the banks of the Yarinacocha lagoon are not aware of the impact of water pollution. Recommendations: It is recommended that public and private organizations can implement programs focused on tourism development, for this it is important that they work directly with the people living on the Yarinacocha Lagoon, owners of establishments and others; Either by giving talks about awareness about water care.
\end{abstract}

Key words: Influence, pollution, development, tourism, stagnation, sustainability. 


\section{Rafael A. Garay Argandoña \\ Docente de la EAP de la Facultad de Ciencias Empresariales y Educación - Universidad Alas Peruanas. \\ Pierre Inuma Rodríguez \\ Estudiante de la EAP de Turismo, Hotelería y Gastronomía - Universidad Alas Peruanas.}

\section{INTRODUCCIÓN}

El presente trabajo de investigación titulado: contaminación de las balsas y restaurantes flotantes en la laguna de Yarinacocha y cómo afecta al desarrollo turístico sostenible. Tiene como propósito fundamental determinar el impacto que tiene la contaminación de las aguas en la Laguna de Yarinacocha por parte de las balsas y restaurantes flotantes y como esto influye en el estancamiento del desarrollo sostenible en el sector turismo, ya que el problema en estudio se viene observando desde hace varios años.

Pérez E., (2000). Presencia del Problema de la Contaminación de las Aguas en el Mundo. “Al nivel mundial el problema de la contaminación de las aguas se agudiza cada día; los agentes transportados por el agua son la principal causa de muertes y transmisión de enfermedades en los llamados países emergentes.

Sin duda los resultados de esta labor investigativa, además de permitirme obtener el título profesional, construirá una fuente de información, muy útil para resolver los problemas que imposibilitan el desarrollo turístico en la ciudad de Pucallpa de manera más eficiente y aprovechando los recursos de modo sostenible, en donde todos los actores salgan beneficiados.

Peck y Lepie (1992) Desarrollo Turístico. Consideran también tres modelos de desarrollo turístico, de crecimiento rápido, crecimiento lento y desarrollo transitorio. El crecimiento rápido se da "cuando las corporaciones adquieren grandes terrenos, y a continuación operan la subdivisión el conocimiento sobre la de los mismos para iniciar la construcción”, y la población se mantiene alejada de los beneficios del turismo.

El presente trabajo de investigación se realizó con la finalidad de conocer los factores que imposibilitan el desarrollo turístico sostenible en el Distrito de Yarinacocha, Región Ucayali- 2016 en el ámbito económico; porque nos dará el resultado de saber cuán importantes es saber manejar el turismo en la laguna de Yarinacocha para un crecimiento económico, social; porque ayudará a saber cuánto ha mejorado la calidad de vida de las 
personas, político; porque veremos en qué medida las instituciones públicas ayudan a la conservación y preservación de los recursos naturales, si existe reglamentos y normas que protejan este lugar turístico y, de Salud; porque se dará a conocer los riesgos y enfermedades que se pueden contraer a causa de entrar en contacto con el agua de la laguna de Yarinacocha. Para ello se desea lograr que las balsas y restaurantes flotantes puedan adaptarse a un modelo de infraestructura adecuada respecto a servicios higiénicos, la cual ayude a la conservación y un manejo responsable del recurso.

Para la realización del siguiente trabajo de investigación la estructura que se utilizó es la siguiente: Capítulo 1: Planteamiento del problema, Capítulo 2: Marco teórico, Capítulo 3: Metodología, Capítulo 4: Conclusiones y recomendaciones, Anexos. Espero haber cumplido con todos los requisitos metodológicos y procedimentales que comprende la investigación científica.

\section{MATERIALES Y MÉTODOS}

La investigación se llevó a cabo utilizando la metodología explicativa, se realizó en el Distrito de Yarinacocha, específicamente alrededor de la laguna de Yarinacocha, que serán desarrollados en puntos estratégicos en donde se concentra el mayor flujo de visitantes, establecimientos comerciales, y pobladores. Para ello se trabajó con una muestra de 50 turistas nacionales, los cuales eran abordados al momento de llegar a la rivera de la Laguna de Yarinacocha.

La presente investigación es de tipo explicativa. Se puede decir que esta clasificación usa como criterio lo que se pretende con la investigación, sea explorar un área no estudiada antes, describir una situación o pretender una explicación del mismo. "Pretende establecer las causas de los eventos, sucesos o fenómenos que se estudian”, Hernández, et al., (2003, p.124).

El diseño utilizado ha sido el transversal, el cual Hernández Fernández y batista (2006) determinan a los diseños de investigación transversal como aquellos donde se recolectan los datos en un solo momento, en un tiempo único. Su propósito es describir variables y analizar su incidencia e interrelación en un momento dado. En la presente investigación se utilizará un diseño que se orienta al grado de influencia que tiene la contaminación de las balsas y restaurantes flotantes en la laguna de Yarinacocha con respecto al Desarrollo Turístico Sostenible.

La técnica empleada para este trabajo de investigación fue la encuesta por medio de un 
cuestionario para evaluar las percepciones que tienen los turistas al visitar la Laguna de Yarinacocha. Los datos fueron recopilados y analizados en el paquete estadístico o software el SPSS versión 21.

\section{RESULTADOS}

Se puede concluir que el $36 \%$ de los encuestados tiene una impresión regular de la laguna de Yarinacocha, de igual forma un 26\% dice que la impresión que llegan a tener es muy buena, así mismo un $24 \%$ asegura que es muy buena. Mientras de otro lado tenemos un $8 \%$ y $6 \%$ que considera que es muy mala y mala la impresión que tienen respectivamente. (Gráf. 1)

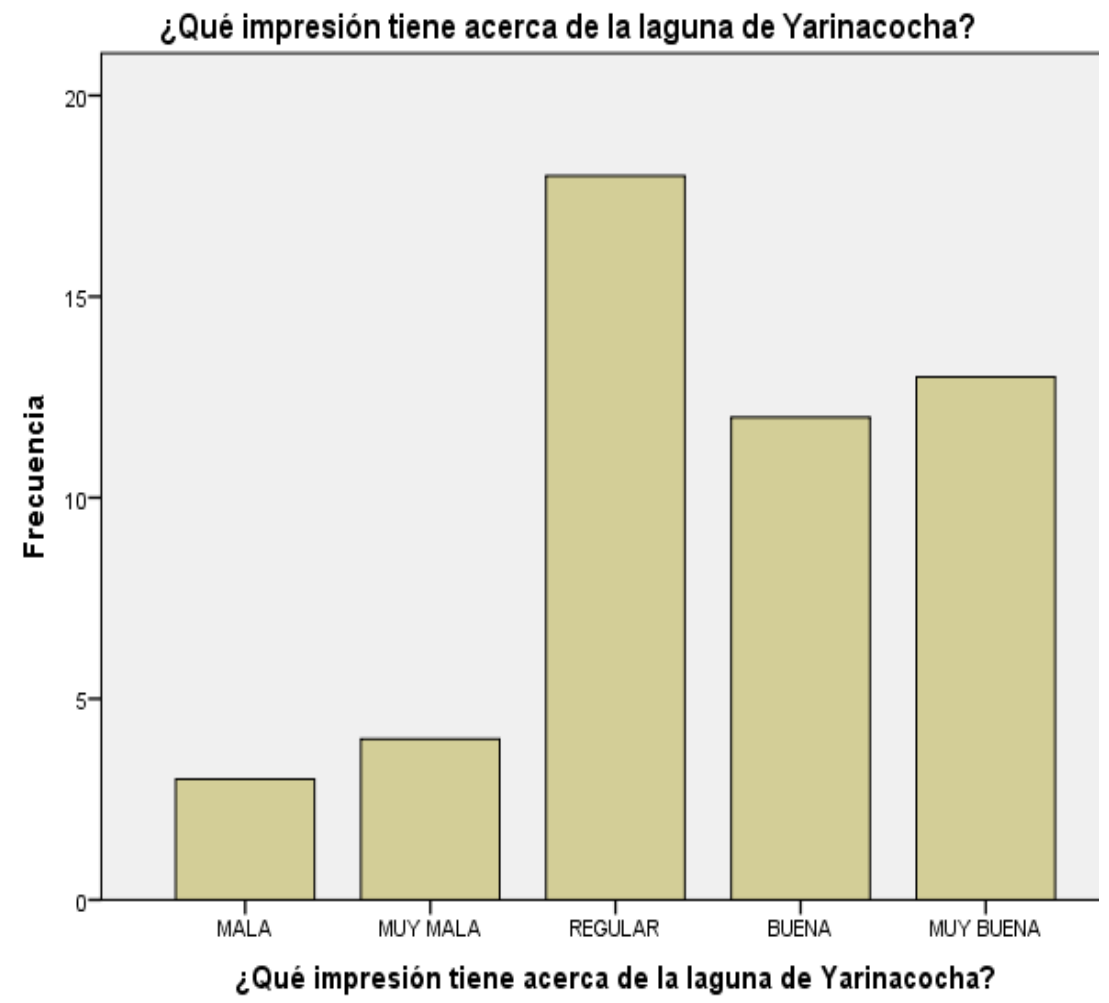

\section{DISCUSIÓN}

Como se ha ido mencionando anteriormente, el estancamiento del desarrollo turístico en la Laguna de Yarinacocha es un problema que ya se viene presentando por muchos años; solo que actualmente aglomeración de las balsas y restaurantes flotantes que se encuentran sobre sus aguas.

Buschmann A.H. 2001. Impacto ambiental de la acuicultura. El estado de la investigación en Chile y el Mundo. Registro de Problemas Públicos N4. Terram Publicación. 67 pp.es. Usualmente, las actividades humanas 
producen cambios en los ecosistemas, los que, muchas veces, generan efectos adversos en el medio ambiente. En ese contexto la acuicultura, al igual que otras actividades económicas, usa y transforma los recursos en productos con un valor económico y social.

Chauveau, (2004) Poner fin al agotamiento y destrucción de recursos naturales. Pero no se trata sólo de las aguas subterráneas: se ha tomado tanta agua de los ríos que, en algunos casos, su caudal ha disminuido drásticamente y apenas llega a su desembocadura, lo cual acaba produciendo irreversibles alteraciones ecológicas: pensemos que muchos peces desovan en el agua dulce que los ríos

\section{CONCLUSIONES}

Se concluye que el posicionamiento de las balsas y restaurantes flotantes dentro de la Laguna de Yarinacocha, afectan significativamente con el desarrollo turístico del mismo, esto imposibilita que pueda mejorar el interés en los posibles visitantes, además que es un factor clave para el desarrollo en otros aspectos que tiene una sociedad como son el económico, político, social y cultural; por la forma en como se ha desarrollado el trabajo además se puede concluir que las personas que habitan en la rivera de la laguna de Yarinacocha no son conscientes del impacto que puede generar la contaminación del agua; además de resaltar introducen en el mar y que muchas especies precisan de los nutrientes que esas aguas acarrean.

El resultado del estudio concuerda con Buschmann y Chauveau, ya que podemos constatar que la Laguna de Yarinacocha verdaderamente se está degradando a razón de que el hombre realiza actividades como el botar desechos, aguas servidas y otros componentes perjudiciales dentro de la laguna y esto como consecuencia puede afectar en la llegada de los visitantes; las cuales tienen que ver con el ámbito económico y social.

que para que pueda existir una mejora de lo que son las buenas prácticas y manejo de recursos ellos aún no han recibido las capacitaciones correspondientes y las herramientas que se necesitan para ponerlos en práctica cada día; así ello se vuelva un hábito.

\section{RECOMENDACIONES}

Se recomienda que las organizaciones públicas y privadas puedan implementar programas enfocados al desarrollo turístico, para ello es importante que trabajen directamente con las personas que viven en la rivera de la Laguna de Yarinacocha, dueños 
de establecimientos y otros; ya sea brindando charlas sobre la concientización sobre el cuidado del Agua.

A los establecimientos que se encuentran sobre la laguna se les recomienda que puedan mejorar la infraestructura de sus instalaciones, adecuarlas a sus necesidades y así contribuir con la preservación del agua. Es importante además que se realice anuncios publicitarios fomentando el conocimiento sobre la importancia que tiene el agua en nuestras vidas, así también se considera que se puedan implementar en la rivera de la laguna de Yarinacocha tachos de basura, y entregar bolsas plásticas a los visitantes para que depositen su basura en ella durante su visita.

\section{REFERENCIAS BIBLIOGRÁFICAS}

1. Buschmann A.H. (2001). Impacto ambiental de la acuicultura. El estado de la investigación en Chile y el Mundo. Registro de Problemas Públicos $\mathrm{N}^{\circ} 4$. Terram Publicación. 67 pp.es. http://www.cetmar.org/DOCUMENTAC ION/dyp/ImpactoChileacuicultura.pdf
2. Chauveau. (2004) Poner fin al agotamiento y destrucción de recursos naturales.

https://www.google.com.pe/?gfe_rd=cr\& ei=RCEmWP7oOa6w8wfLlb_ICA\#

3. Hernández Fernández y batista. (2006). Diseño

deinvestigacióntransversal.https://www.3 A\%2F\%2Fmetodologia02.blogspot.com $\% 2 \mathrm{Fp} \% 2$ Foperacionalizacion-devariables.html

4. Hernández. (2003), p.124.Pretende establecer las causas de los eventos, sucesos $\quad$ o fenómenos queseestudian.https://www.2Fmetodolog ia02.blogspot.com\%2Fp\%2Foperacionali zacion-de variables.html.

5. Peck y Lepie. (1992). Desarrollo Turístico. http://www.ugr.es/ pwlac/G20_17Angel _Acuna_Delgado.html

6. Pérez E. (2000) Presencia del Problema de la Contaminación de las Aguas en el Mundo, (p. 271).http://www.scielo.org.co/pdf/pml/v 7n2/v7n2a05.pdf 